\title{
Fatal Disseminated Tuberculosis during Treatment with Ruxolitinib Plus Prednisolone in a Patient with Primary Myelofibrosis: A Case Report and Review of the Literature
}

\author{
Yasuhiro Tsukamoto ${ }^{1,2}$, Junichi Kiyasu ${ }^{1,3}$, Mariko Tsuda ${ }^{1,2}$, Motohiko Ikeda ${ }^{1}$, \\ Motoaki Shiratsuchi ${ }^{2}$, Yoshihiro Ogawa ${ }^{2}$ and Yuji Yufu ${ }^{1}$
}

\begin{abstract}
:
A 73-year-old man with primary myelofibrosis (PMF) was being treated with hydroxyurea, which was changed to ruxolitinib treatment because of worsening constitutional symptoms. Although ruxolitinib rapidly induced relief, he developed a high-grade fever. A comprehensive fever work-up found no apparent cause of the fever, except for PMF. Therefore, we increased the dose of ruxolitinib and added prednisolone, which was gradually withdrawn with resolution of the fever. However, the patient subsequently developed disseminated tuberculosis and died eight months after initiation of ruxolitinib. Our case highlights the importance of assessing and monitoring the immune status of patients receiving ruxolitinib.
\end{abstract}

Key words: primary myelofibrosis, ruxolitinib, disseminated tuberculosis

(Intern Med 57: 1297-1300, 2018)

(DOI: 10.2169/internalmedicine.9165-17)

\section{Introduction}

Primary myelofibrosis (PMF) is a clonal hematopoietic stem cell disorder resulting from mutations that include the JAK-STAT signaling regulator genes and/or epigenetic regulator genes (1). Allogeneic hematopoietic stem cell transplantation is the only treatment with curative potential for PMF, with other treatment options being palliative and focused on alleviating the constitutional symptoms. Ruxolitinib, a selective inhibitor of $J A K 1$ and $J A K 2$, significantly reduces splenomegaly and relieves constitutional symptoms, which results in an improved quality of life for patients with PMF (2). Although ruxolitinib is a promising and active treatment option, several recent case reports have suggested that patients treated with ruxolitinib might be at risk for various opportunistic infections, including reactivation of tuberculosis (3). However, because of a lack of evidence, there are no criteria for identifying those high-risk patients being treated with ruxolitinib who should be considered for prophylaxis/treatment.
We herein report a patient with PMF who developed fatal disseminated tuberculosis during treatment with ruxolitinib plus prednisolone.

\section{Case Report}

A 73-year-old man was referred to our hospital because of marked splenomegaly ( $6 \mathrm{~cm}$ below the costal margin), thrombocytosis, and an elevated serum lactate dehydrogenase level in April 2013. A bone marrow biopsy showed grade 3 fibrosis with the JAK2 V617F mutation. He was diagnosed with PMF with an intermediate-2 risk score by the Dynamic International Prognostic Scoring System (DIPSS) Plus (4). Because ruxolitinib was not available in Japan at the time, he first received hydroxyurea (HU; $500 \mathrm{mg}$ daily) to alleviate abdominal distension due to splenomegaly, which partially improved soon after the initiation of $\mathrm{HU}$. However, his constitutional symptoms, including anemia, a low-grade fever, night sweats, and abdominal distention (splenomegaly $10 \mathrm{~cm}$ below the costal margin), worsened two years after starting $\mathrm{HU}$.

${ }^{1}$ Department of Hematology, Iizuka Hospital, Japan, ${ }^{2}$ Department of Medicine and Bioregulatory Science, Graduate School of Medical Sciences, Kyushu University, Japan and ${ }^{3}$ Department of Clinical Research Institute, National Kyushu Cancer Center, National Hospital Organization, Japan Received: March 8, 2017; Accepted: September 13, 2017; Advance Publication by J-STAGE: December 27, 2017 Correspondence to Dr. Junichi Kiyasu, jkiyasuh2@aih-net.com 


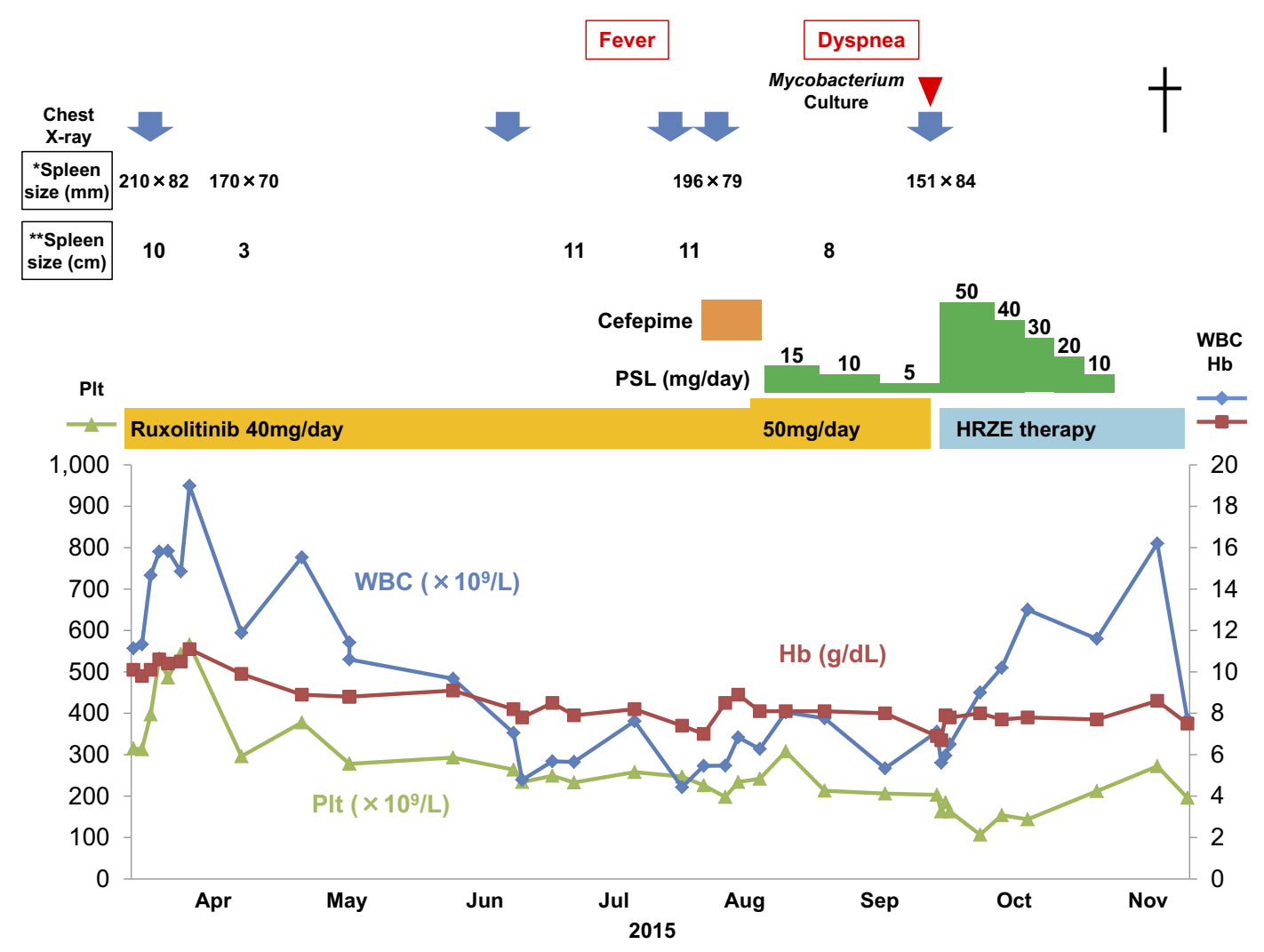

Figure. The clinical course of the patient. Spleen enlargement was evaluated by *abdominal ultrasonography and **palpation (centimeters below the costal margin). PSL: prednisolone, HRZE: isoniazid, rifampin, pyrazinamide, and ethambutol

The patient underwent a diagnostic fever work-up, which included the interferon-gamma release assay [IGRA; TSPOT TB assay (Oxford Immunotec, Oxford, United Kingdom)]. Although the result was positive, other clinical examinations did not reveal obvious signs of active tuberculosis. We did not perform Mycobacterium culture at that time. We changed the patient's therapy to ruxolitinib (20 mg twice daily). Ruxolitinib induced rapid relief of symptoms and reduced his splenomegaly ( $3 \mathrm{~cm}$ below the costal margin). However, 4 months later, the patient's spleen had enlarged to $11 \mathrm{~cm}$ below the costal margin, and he developed a highgrade fever. A diagnostic fever work-up was performed, including conventional culture tests of blood, urine, sputum, and cerebrospinal fluid (Mycobacterium cultures were not performed) and whole-body computed tomography (CT). No other causes of fever except for PMF could be identified. The patient received broad-spectrum antibacterial treatment (cefepime) but remained febrile. We concluded that progression of PMF was responsible for the high-grade fever and increased the dose of ruxolitinib to $25 \mathrm{mg}$ twice daily while adding prednisolone (15 mg daily) to alleviate the patient's constitutional symptoms. The fever abated temporarily, and the prednisolone dose was gradually tapered to $5 \mathrm{mg}$ daily.

Two months later, the patient suddenly developed rapidly progressing dyspnea. Chest CT revealed numerous 2- to 3$\mathrm{mm}$ nodules distributed throughout both lungs. Mycobacterium tuberculosis was isolated from Mycobacterium cultures of sputum, gastric lavage fluid, blood, and urine. Ruxolitinib was immediately discontinued with no cytokine rebound phenomenon. Prednisolone was temporarily increased to 50 mg daily for this critically ill patient with pulmonary tuberculosis and acute respiratory failure (5) and then tapered by $10 \mathrm{mg}$ per week for the treatment of disseminated tuberculosis, without any withdrawal symptoms. The patient was started on an antituberculosis regimen of rifampicin, isoniazid, pyrazinamide, and ethambutol. However, he did not respond to the antituberculosis treatment. Regarding the PMF, the blood cell count was stable, and his constitutional symptoms were relatively mild during the course of the disease. However, his condition gradually worsened, and he died of respiratory failure three months after the diagnosis of reactivated tuberculosis. The clinical course of the patient is summarized in Figure.

\section{Discussion}

In a previous report, patients with myeloproliferative neoplasm (MPN) had an increased risk of tuberculosis infection compared with the normal population (6); furthermore, patients with primary myelofibrosis were clearly at a higher risk for tuberculosis infection than those with other MPNs, such as chronic myelogenous leukemia, polycythemia vera, and essential thrombocythemia. Recently, Polverelli et al. reported risk factors for infections in patients with myelofibro- 
Table. Previous Reports of Disseminated Tuberculosis (Tb) in Patients with Myeloproliferative Neoplasms.

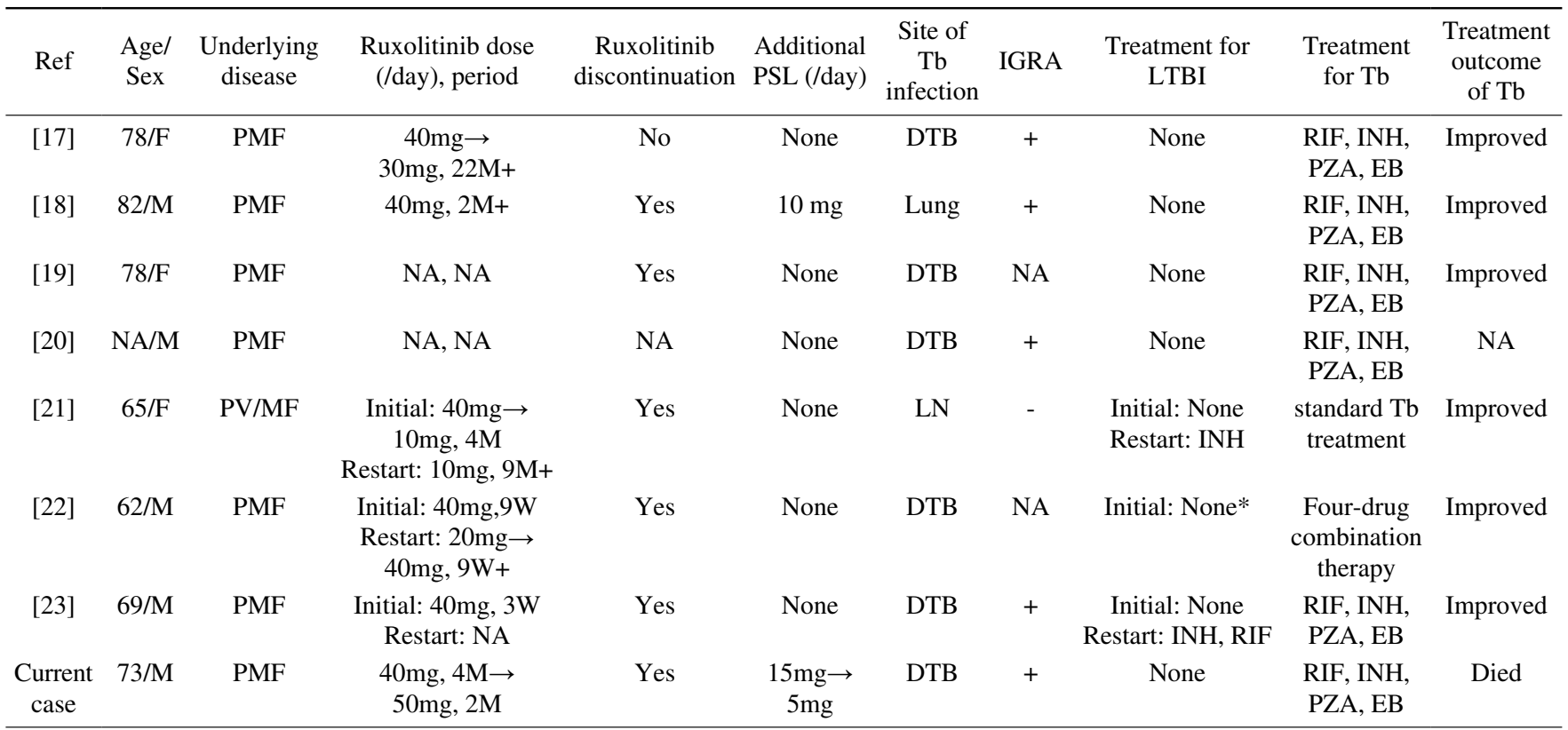

*: During Tb treatment, ruxolitinib was first stopped and then restarted. NA: not available, PMF: primary myelofibrosis, PV: polycythemia vera, MF: myelofibrosis, for the ruxolitinib dose period: M: month, W: week, PSL: prednisolone, DTB: disseminated tuberculosis, LN: lymph node, IGRA: interferon-gamma release assay, LTBI: latent tuberculosis infection, INH: isoniazid, RIF: rifampin, PZA: pyrazinamide, EB: ethambutol

sis in a retrospective multicenter study of 507 patients. In this population, three patients developed tuberculosis infections (two received ruxolitinib treatment), and patients treated with ruxolitinib had a higher cumulative incidence of infections than those not treated with ruxolitinib (7).

According to recent prospective, phase 3, clinical trials (COMFORT-I, COMFORT-II and JUMP) of ruxolitinib, the incidence of infectious diseases was not higher in patients taking ruxolitinib than in those who received placebo or the best available therapy. However, some patients developed opportunistic infections, including reactivated tuberculosis (8-10). Accumulating data have suggested that treatment with ruxolitinib induces several adverse effects associated with its immunosuppressive activity. In addition to reactivated tuberculosis, reports on Cryptococcus neoformans pneumonia, Toxoplasmosis retinitis, Pneumocystis jiroveci pneumonitis, and hepatitis $\mathrm{B}$ virus reactivation in patients treated with ruxolitinib have been published (11-14).

Table summarizes ours and previous case reports on tuberculosis reactivation in patients treated with ruxolitinib. The duration of ruxolitinib administration before tuberculosis reactivation ranged from 3 weeks to 22 months, and most of the patients developed disseminated tuberculosis. Except for our case, screening for latent tuberculosis infection (LTBI) by IGRA was not performed prior to the initiation of ruxolitinib treatment; therefore, treatment for LTBI was not implemented for any of the previous cases. One other patient in addition to our own received low-dose prednisolone together with ruxolitinib. After the reactivation of tuberculosis, ruxolitinib was discontinued in most cases, and all patients received standard antituberculosis therapy. Although the outcome of tuberculosis treatment was generally favorable, the infection in our case was resistant to antibiotics, and the patient's clinical course was dismal.

Although the incidence of life-threatening opportunistic infections among patients treated with ruxolitinib is not very high, these complications do occur. Occasionally, corticosteroids are used to relieve refractory constitutional symptoms and consequently produce a feeling of well-being (15). According to the Centers for Disease Control and Prevention guidelines, patients receiving prednisolone (or its equivalent) at doses greater than $15 \mathrm{mg} /$ day for 2 to 4 weeks are at high risk for tuberculosis reactivation, and treatment for LTBI is recommended (16). In our case, the coexistence of poorly controlled PMF plus ruxolitinib and prednisolone (15 mg/ day with tapering) immunosuppressive therapy might have resulted in a serious degree of immunosuppression and inadequate host immune response to the pathogen. Because a fever and night sweats are common constitutional symptoms not only of PMF but also of tuberculosis, recognizing the onset of tuberculosis reactivation during the course of PMF was difficult. As we had obtained positive IGRA findings before initiating ruxolitinib treatment, we probably should have considered LTBI treatment for this patient, at least when prednisolone was added to his PMF therapy. However, the number of PMF cases with reactivated tuberculosis that have been reported to date is too low to yield definitive conclusions.

In summary, although ruxolitinib is an attractive treatment option for PMF patients with severe constitutional symptoms, the deleterious effects of ruxolitinib should be noted. We should also keep in mind that the signs and symptoms of ruxolitinib-induced opportunistic infections are important for the differential diagnosis of the constitutional symptoms 
of PMF. Although evidence is not yet sufficient to quantify the risk of opportunistic infections in ruxolitinib-treated patients, anti-infective prophylaxis/treatment might be necessary, especially in select patients with risk factors (e.g. based on the status of the underlying disease and concomitant use of other immunosuppressive agents). The appropriate assessment and monitoring of the immune status before and during ruxolitinib treatment may be a key strategy in the successful management of patients with PMF.

The authors state that they have no Conflict of Interest (COI).

\section{References}

1. Tefferi A, Vainchenker W. Myeloproliferative neoplasms: molecular pathophysiology, essential clinical understanding, and treatment strategies. J Clin Oncol 29: 573-582, 2011.

2. Vannucchi AM, Kantarjian HM, Kiladjian JJ, et al. A pooled analysis of overall survival in COMFORT-I and COMFORT-II, 2 randomized phase III trials of ruxolitinib for the treatment of myelofibrosis. Haematologica 100: 1139-1145, 2015.

3. Heine A, Brossart P, Wolf D. Ruxolitinib is a potent immunosuppressive compound: is it time for anti-infective prophylaxis? Blood 122: 3843-3844, 2013.

4. Gangat N, Caramazza D, Vaidya R, et al. DIPSS plus: a refined Dynamic International Prognostic Scoring System for primary myelofibrosis that incorporates prognostic information from karyotype, platelet count, and transfusion status. J Clin Oncol 29: 392397, 2011.

5. Yang JY, Han M, Koh Y, et al. Effects of corticosteroids on critically ill pulmonary tuberculosis patients with acute respiratory failure: a propensity analysis of mortality. Clin Infect Dis 63: 1449-1455, 2016.

6. Tassies D, Cervantes F, Nicolas JM, Feliu E, Soriano E, Rozman C. Tuberculosis in chronic myeloproliferative syndromes: its incidence and principle characteristics in a series of 562 patients. Med Clin (Barc) 96: 321-323, 1991.

7. Polverelli N, Breccia M, Benevolo G, et al. Risk factors for infections in myelofibrosis: role of disease status and treatment. A multicenter study of 507 patients. Am J Hematol 92: 37-41, 2017.

8. Verstovsek S, Mesa RA, Gotlib J, et al. A double-blind, placebocontrolled trial of ruxolitinib for myelofibrosis. N Engl J Med 366: 799-807, 2012.

9. Harrison C, Kiladjian JJ, Al-Ali HK, et al. JAK inhibition with ruxolitinib versus best available therapy for myelofibrosis. N Engl J Med 366: 787-798, 2012.

10. Al-Ali HK, Griesshammer M, le Coutre P, et al. Safety and effi- cacy of ruxolitinib in an open-label, multicenter, single-arm phase $3 b$ expanded-access study in patients with myelofibrosis: a snapshot of 1144 patients in the JUMP trial. Haematologica 101: 1065-1073, 2016.

11. Lee SC, Feenstra J, Georghiou PR. Pneumocystis jiroveci pneumonitis complicating ruxolitinib therapy. BMJ 2014 (Epub ahead of print).

12. Wysham NG, Sullivan DR, Allada G. An opportunistic infection associated with ruxolitinib, a novel janus kinase 1,2 inhibitor. Chest 143: 1478-1479, 2013.

13. Caocci G, Murgia F, Podda L. Reactivation of hepatitis B virus infection following ruxolitinib treatment in a patient with myelofibrosis. Leukemia 28: 225-227, 2014.

14. Goldberg RA, Reichel E, Oshry LJ. Bilateral toxoplasmosis retinitis associated with ruxolitinib. N Engl J Med 369: 681-683, 2013.

15. Vannucchi AM. Management of myelofibrosis. ASH Education Program Book 2011: 222-230, 2011.

16. Targeted tuberculin testing and treatment of latent tuberculosis infection. This official statement of the American Thoracic Society was adopted by the ATS Board of Directors, July 1999. This is a Joint Statement of the American Thoracic Society (ATS) and the Centers for Disease Control and Prevention (CDC). This statement was endorsed by the Council of the Infectious Diseases Society of America. (IDSA), September 1999, and the sections of this statement. Am J Respir Crit Care Med 161: 221-247, 2000.

17. Branco B, Metsu D, Dutertre M, et al. Use of rifampin for treatment of disseminated tuberculosis in a patient with primary myelofibrosis on ruxolitinib. Ann Hematol 95: 1207-1209, 2016.

18. Chen $\mathrm{YH}$, Lee $\mathrm{CH}$, Pei SN. Pulmonary tuberculosis reactivation following ruxolitinib treatment in a patient with primary myelofibrosis. Leuk Lymphoma 56: 1528-1529, 2015.

19. Shamil E, Cunningham D, Wong BL. Ruxolitinib associated tuberculosis presenting as a neck lump. Case Rep in Infect dis 2015: 284168, 2015.

20. Colomba C, Rubino R, Siracusa L, et al. Disseminated tuberculosis in a patient treated with a JAK2 selective inhibitor: a case report. BMC Res Notes 5: 552, 2012.

21. Palandri F, Polverelli N, Catani L. Ruxolitinib-associated tuberculosis: a case of successful ruxolitinib rechallenge. Ann Hematol 94: 519-520, 2015.

22. Hopman RK, Lawrence SJ, Oh ST. Disseminated tuberculosis associated with ruxolitinib. Leukemia 28: 1750-1751, 2014.

23. Abidi MZ, Haque J, Varma $\mathrm{P}$, et al. Reactivation of pulmonary tuberculosis following treatment of myelofibrosis with ruxolitinib. Case Rep Hematol 2016: 2389038, 2016.

The Internal Medicine is an Open Access article distributed under the Creative Commons Attribution-NonCommercial-NoDerivatives 4.0 International License. To view the details of this license, please visit (https://creativecommons.org/licenses/ by-nc-nd/4.0/).

(C) 2018 The Japanese Society of Internal Medicine Intern Med 57: 1297-1300, 2018 